\title{
Relationship between thyroid-stimulating hormone and blood pressure in the middle-aged and elderly population
}

Wei-Xia Jian' ${ }^{1}$, MD, PhD, Jie Jin ${ }^{1}$, MD, Li Qin ${ }^{1}$, MD, Wen-Jun Fang ${ }^{1}$, MD, Xue-Ru $\underline{\text { Chen }}^{1}$, MD, Han-Bei $\underline{\text { Chen }}^{1}$, MD, Qing $\underline{S u}^{1}$, MD, PhD, Hui-Li Xing ${ }^{1,2}$, MD

INTRODUCTION Hypothyroidism and subclinical hypothyroidism may be associated with hypertension and metabolic syndrome. The aim of this study was to investigate the relationship between thyroid-stimulating hormone (TSH) and blood pressure, as well as the relationship between thyroid function and insulin resistance in middle-aged and elderly Chinese.

METHODS This was a cross-sectional, community-based study. Serum TSH, fasting glucose and insulin were measured in 2,988 subjects aged 35-80 years. Logistic regression analysis was used to identify the risk factors for hypertension. Analysis of variance and multiple linear regression analysis were performed to characterise the relationship among TSH, insulin resistance and blood pressure.

RESULTS Higher serum TSH concentration was found to be an independent risk factor for hypertension in females (odds ratio 1.4, 95\% confidence interval 1.02-1.93; p-value $=0.039$ ). The female group with subclinical hypothyroidism and high normal TSH (2.5-4.8 mIU/L) were more susceptible to high blood pressure than those with low normal TSH $(0.3-2.5 \mathrm{mIU} / \mathrm{L})$ ( $\mathrm{p}$-value < 0.05). After adjustment for waist-hip ratio and body mass index, neither the correlation between blood pressure and homeostasis model assessment of insulin resistance (HOMA-IR) nor the correlation between TSH and HOMA-IR were found to be significant in this study.

CONCLUSION This study provides evidence that both subclinical hypothyroidism and high normal TSH are independent risk factors for hypertension in middle-aged and elderly Chinese women.

Keywords: hypertension, hypothyroidism, insulin resistance, thyrotropin

\section{INTRODUCTION}

Patients with hypothyroidism often have concurrent diastolic hypertension and hyperlipidaemia, and are hence susceptible to the development of coronary heart disease. Previous studies have shown that hypertension occurs in $20 \%-40 \%$ of hypothyroid patients. ${ }^{(1,2)}$ However, the association between hypertension and subclinical hypothyroidism or high normal thyroid-stimulating hormone (TSH), also known as thyrotropin, remains controversial. A large population-based study performed on the habitants of Norway showed a positive linear association between the normal reference range of TSH and blood pressure. ${ }^{(3)}$ Ruhla et al found that a high normal TSH was associated with a 1.7-fold increased prevalence of metabolic syndrome, ${ }^{(4)}$ and since hypertension and insulin resistance are important symptoms of metabolic syndrome, the authors suggested that high normal TSH may be a risk factor for hypertension and insulin resistance. A possible mechanism that could explain the association of increased TSH with increased blood pressure is endothelial dysfunction resulting from a reduction in nitric oxide availability, leading to increased systemic vascular resistance independent of hyperlipidaemia. ${ }^{(5)}$ It was also revealed that endothelial dysfunction in subclinical hypothyroidism could be partially reversed by levothyroxine supplementation..$^{(5)}$ However, even though systemic vascular resistance is increased, blood pressure may remain nearly stable in subclinical hypothyroidism because of diminished cardiac output. Walsh et al found that subclinical hypothyroidism was not associated with hypertension in a Western Australian population. ${ }^{(6)}$ The inconsistent results on the relationship of high normal TSH level with blood pressure warrant further investigations in large populations.

In the present study, we explored the relationship between subclinical hypothyroidism or high normal TSH and blood pressure in a population. The study population was originally recruited during a health survey on the prevalence of thyroid disease and diabetes mellitus in the district of Baoshan in Shanghai, China. Since existing research conducted in other populations have suggested that insulin resistance may also be associated with blood pressure, ${ }^{(7,8)}$ we investigated the relationships among serum TSH level, insulin resistance and blood pressure.

\section{METHODS}

Individuals recruited in this study were local residents of the Baoshan district in Shanghai, China. Since hypertension and

${ }_{1}^{1}$ Department of Endocrinology, Xinhua Hospital, Shanghai Jiaotong University School of Medicine, ${ }^{2}$ Department of Endocrinology, Shanghai Yangsi Hospital, Shanghai, China

Correspondence: Prof Hui-Li Xing, Professor, Department of Endocrinology, Xinhua Hospital, Shanghai Jiaotong University School of Medicine, 1665 Kongjiang Road, Shanghai 200092, China.dr_huilixing@126.com 
metabolic syndrome are major health problems in the aged population, and serum TSH is affected by age, ${ }^{(9)}$ we studied middle-aged and elderly adults aged 35-80 years. Written informed consent was obtained from all individuals participating in this study. The recruited individuals also completed a health and lifestyle questionnaire. To avoid the influence of confounding factors, the following were excluded from the study: (a) individuals with known severe renal, liver, heart or brain diseases; (b) individuals with a history of thyroid disease or diabetes mellitus; and (c) individuals who were taking medicines that may influence thyroid function and blood glucose concentration. The diagnostic criteria for hypertension were systolic blood pressure (SBP) and diastolic blood pressure (DBP) above $140 \mathrm{mmHg}$ and $90 \mathrm{mmHg}$, respectively, as well as current or previous use of antihypertensive medication. Individuals with treated hypertension were excluded from the quantitative analyses of blood pressure, but included in the analyses of hypertension prevalence. The questionnaire, physical examination and biochemical tests were completed in a total of 2,988 residents. Since the normal TSH reference range is still debatable, and a narrower reference range has been suggested, ${ }^{(10)}$ the recruited individuals were divided into three subgroups: (a) those with low normal TSH $(0.3-2.5 \mathrm{mlU} / \mathrm{L})$; (b) those with high normal TSH (2.5-4.8 $\mathrm{mlU} / \mathrm{L})$; and (c) those with subclinical hypothyroidism (TSH $>4.8 \mathrm{mIU} / \mathrm{L})$.

Body height, weight, waist circumference (i.e. narrowest circumference between the lower coastal margin and iliac crest), hip circumference (i.e. maximum circumference at the level of the femoral trochanters) and blood pressure were measured. Blood pressure was measured twice using a standard mercury sphygmomanometer, after at least five minutes of rest and in a seated position. The mean of the two measurements was used for analysis. Mean arterial pressure (MAP) was calculated using the formula: $\mathrm{MAP}=\mathrm{DBP}+1 / 3(\mathrm{SBP}-\mathrm{DBP})$. Body mass index (BMI) was calculated as weight $(\mathrm{kg})$ divided by the square of height $\left(\mathrm{m}^{2}\right)$, and waist-hip ratio (WHR) was calculated as waist circumference $(\mathrm{cm})$ divided by hip circumference $(\mathrm{cm})$.

Blood samples were collected for biochemical analysis after overnight fasting for at least ten hours, and frozen in aliquots at $-70^{\circ} \mathrm{C}$ within four hours. All biochemical assays were performed in a central laboratory in Shanghai's Xinhua Hospital, and serum TSH was determined using the Bayer CENTAUR chemiluminescence analyser (Bayer Inc, Leverkusen, Germany). The normal reference of serum TSH was $0.3-4.8 \mathrm{mIU} / \mathrm{L}$. After serum TSH was determined in all individuals, serum free triiodothyronine and free thyroxine were tested in those with serum TSH levels $>4.8 \mathrm{mIU} / \mathrm{L}$. Individuals with serum $\mathrm{TSH}$ levels $<0.3 \mathrm{mIU} / \mathrm{L}$ and those with clinical hypothyroidism were excluded from the study. Fasting plasma glucose (FPG) was determined using an automated glucose oxidase method, and serum insulin was measured using Insulin RIA DSL1600 competitive radioimmunoassay (Diagnostic Systems
Laboratories Inc, Webster, TX, USA). The homeostasis model assessment of insulin resistance (HOMA-IR) was calculated using the following formula: FPG $(\mathrm{mmol} / \mathrm{L}) \times$ fasting serum insulin $(\mathrm{mlU} / \mathrm{L}) / 22.5$.

Statistical analyses were done using the Statistical Package for the Social Sciences version 13 (SPSS Inc, Chicago, IL, USA). All reported $p$-values are based on two-sided tests, and the nominal significance level was set at 0.05. For descriptive analyses, mean and standard deviation (SD) values were used for continuous variables, while counts and percentages were used for discrete variables. Normal distribution of all quantitative traits was tested using the Kolmogorov D normal test. In the present study, both serum TSH and HOMA-IR values were logarithmically transformed to a normal distribution for statistical analyses. Unpaired $t$-test was used to examine the difference in baseline characteristics between males and females. Logistic regression analysis was performed to detect risk factors for hypertension. Since the relationship between serum TSH and blood pressure may be inconsistent in the male and female groups, ${ }^{(11)}$ logistic regression analyses were also performed in male and female subgroups. Analysis of variance (ANOVA) was performed to examine the association between blood pressure and different TSH ranges in females and males. Spearman correlation analysis and multiple linear regression analysis were used to analyse the relationships among HOMA-IR, TSH and blood pressure levels.

\section{RESULTS}

Of the 2,988 residents investigated, 2,344 individuals were included in the statistical analysis. The baseline characteristics of the 2,344 individuals are shown in Table I. As shown in Table I, age, BMI, SBP, DBP, WHR, logarithmically transformed TSH (logTSH) values and percentage of hypertension, smoking and drinking were significantly different between the female and male groups. There were no significant differences in FPG, logarithmically transformed HOMA-IR (logHOMA-IR) and percentage of history of hyperlipidaemia between the two groups.

Using multiple logistic regression analysis, the following was found to be independent risk factors for hypertension: (a) higher logTSH (odds ratio [OR] 1.40, 95\% confidence interval [CI] $1.02-1.93$; $\mathrm{p}=0.039$ ); (b) male gender $(\mathrm{OR} 1.73,95 \% \mathrm{Cl}$ 1.31-2.29; $\mathrm{p}<0.001)$; (c) older age (OR 1.04, 95\% Cl 1.03-1.05; $\mathrm{p}<0.001$ ); (d) BMI (OR 1.09, 95\% Cl 1.06-1.12; $\mathrm{p}<0.001$ ); (e) WHR (OR 14.04, 95\% Cl 2.76-71.55; p < 0.001); (f) drinking (OR 1.34, 95\% Cl 1.01-1.78; $\mathrm{p}=0.040)$; and (g) history of hyperlipidaemia (OR 1.63, 95\% Cl 1.18-2.26; $\mathrm{p}=0.003)$. Unexpectedly, the insulin resistance index, HOMA-IR, was not found to be significantly associated with hypertension. However, using Spearman correlation analysis, logHOMA-IR was found to be significantly correlated with blood pressure level, and the correlation coefficients for 
Table I. Baseline characteristics of the subjects $(n=2,344)$.

\begin{tabular}{lccr}
\hline Parameter & \multicolumn{2}{c}{ Mean \pm SD } & p-value \\
\cline { 2 - 3 } & $\begin{array}{c}\text { Female } \\
(\mathbf{n}=\mathbf{1 , 6 2 3})\end{array}$ & $\begin{array}{c}\text { Male } \\
(\mathbf{n}=\mathbf{7 2 1})\end{array}$ & \\
\hline Age $(\mathrm{yrs})$ & $54.6 \pm 11.7$ & $57.3 \pm 10.9$ & $<0.01$ \\
BMI $\left(\mathrm{kg} / \mathrm{cm}^{2}\right)$ & $24.03 \pm 3.45$ & $24.35 \pm 3.41$ & 0.04 \\
SBP $(\mathrm{mmHg})$ & $123 \pm 17$ & $129 \pm 16$ & $<0.01$ \\
DBP $(\mathrm{mmHg})$ & $78 \pm 10$ & $83 \pm 10$ & $<0.01$ \\
WHR & $0.85 \pm 0.06$ & $0.88 \pm 0.06$ & $<0.01$ \\
FPG $(\mathrm{mmol} / \mathrm{L})$ & $4.71 \pm 0.81$ & $4.73 \pm 0.83$ & 0.51 \\
logHOMA-IR & $0.32 \pm 0.29$ & $0.30 \pm 0.31$ & 0.22 \\
logTSH & $0.35 \pm 0.29$ & $0.22 \pm 0.28$ & $<0.01$ \\
Smoking (\%) & 1.8 & 61.0 & $<0.01$ \\
Drinking (\%) & 4.1 & 47.0 & $<0.01$ \\
History of & 7.6 & 8.7 & 0.34 \\
hyperlipidaemia (\%) & & & \\
Hypertension (\%) & 23.5 & 30.7 & $<0.01$ \\
\hline
\end{tabular}

BMI: body mass index; DBP: diastolic blood pressure; FPG: fasting plasma glucose; logHOMA-IR: logarithmically transformed homeostasis model assessment of insulin resistance; logTSH: logarithmically transformed thyroid-stimulating hormone; SBP: systolic blood pressure; SD: standard deviation; WHR: waist-hip ratio

Table II. Logistic regression analysis results of risk factors for hypertension.

\begin{tabular}{lccc}
\hline Parameter & OR value & $\mathbf{9 5 \%} \mathbf{C l}$ & p-value \\
\hline logTSH & 1.40 & $1.02-1.93$ & 0.039 \\
logHOMA-IR & 1.22 & $0.89-1.68$ & 0.22 \\
Gender & 1.73 & $1.31-2.29$ & $<0.001$ \\
Age & 1.04 & $1.03-1.05$ & $<0.001$ \\
BMI & 1.09 & $1.06-1.12$ & $<0.001$ \\
WHR & 14.04 & $2.76-71.55$ & $<0.001$ \\
Smoking & 0.72 & $0.53-0.98$ & 0.056 \\
Drinking & 1.34 & $1.01-1.78$ & 0.040 \\
History of hyperlipidaemia & 1.63 & $1.18-2.26$ & 0.003 \\
\hline
\end{tabular}

BMI: body mass index; $\mathrm{Cl}$ : confidence interval; logHOMA-IR: logarithmically transformed homeostasis model assessment of insulin resistance; logTSH: logarithmically transformed thyroid-stimulating hormone; OR: odds ratio; WHR: waist-hip ratio

SBP, DBP and MAP were $0.18,0.12$ and $0.16(p<0.001)$, respectively. The significant correlation between HOMA-IR and blood pressure became non-significant when anthropometric indices, including BMI and WHR as covariances, were taken into account in the multiple linear regression analysis (data not shown).

Serum TSH levels in the female group were found to be significantly higher than those in the male group, suggesting that gender may influence serum TSH levels. We thus performed further logistic regression analysis to separately examine the hypertension risk factors in the female and male groups. After adjustment for the effects of age, BMI, WHR, smoking, drinking and history of hyperlipidaemia, TSH concentration was found to be an independent risk factor for hypertension in females (OR 1.27, 95\% Cl 1.09-1.49; $\mathrm{p}=0.003)$, but not in males (OR 1.25, 95\% Cl 0.94-1.67; $p=0.131$ ) (Table III). The results of the ANOVA analysis in the female subgroup are shown in Table IV; SBP, DBP and MAP were significantly higher in females with subclinical hypothyroidism than those with euthyroidism. Furthermore, we found that SBP ( $p=0.004)$, DBP $(p=0.024)$ and MAP $(p=0.003)$ were significantly increased, with higher TSH concentration in the female group (Fig. 1), but not in the male group (data not shown).

Using Spearman correlation analysis, significant association was found between TSH and HOMA-IR $(r=0.05 ; p=0.017)$. However, after adjusting for the confounding effects of BMI and WHR on insulin resistance, multiple linear regression analysis showed that TSH was not significantly associated with HOMA-IR. The significant independent factors for variation of HOMA-IR were drinking $(p=0.001)$, BMI $(p<0.001)$, WHR $(p=0.017)$ and SBP $(p<0.007)$.

\section{DISCUSSION}

In this cross-sectional study, we found that raised serum TSH concentration was a significant independent risk factor for hypertension in the middle-aged and elderly female population. The female group with high normal TSH was more susceptible to hypertension than the group with low normal $\mathrm{TSH}$. We also found that higher BMI and WHR, old age, male gender, history of hyperlipidaemia and drinking were independent risk factors for hypertension. Surprisingly, after adjusting for the effects of the covariates WHR and BMI, no significant correlations were found between blood pressure and HOMA-IR, or between TSH and HOMA-IR.

The effects of thyroid hormone on blood pressure regulation and the cardiovascular system are well recognised. The effects of hypothyroidism on the cardiovascular system include increased systemic vascular resistance, decreased cardiac output and narrowed pulse pressure. Increased sympathetic activity and adrenal activation in hypothyroidism may also contribute to the development of arterial hypertension. ${ }^{(12)}$ As even a mild degree of hypothyroidism may have health consequences, the upper limit of the normal range of serum TSH has decreased over the years. ${ }^{(10,13)}$ Individuals with TSH values in the high normal range have endothelial dysfunction and decreased endothelium-dependent vasodilation. ${ }^{(14)}$ Therefore, thyroid function may play a role in the pathogenesis of hypertension in euthyroid individuals. The results of our study are in accordance with some previous studies, ${ }^{(3,15,16)}$ supporting the viewpoint that subclinical hypothyroidism and high normal TSH are correlated with higher blood pressure in females. However, a study conducted in an Italian population did not find any significant correlation between TSH and blood pressure. ${ }^{(17)}$ In a communitybased study, Walsh et al also suggested that subclinical hypothyroidism was not associated with hypertension in a Western Australian population. ${ }^{(6)}$ While the exact causes of this discrepancy are not clear, we note that these studies enrolled individuals based on different criteria. While the individuals 
Table III. Logistic regression analysis results of hypertension according to TSH ranges in the female and male groups.

\begin{tabular}{|c|c|c|c|c|}
\hline \multirow[t]{2}{*}{ Gender } & \multicolumn{3}{|c|}{ No. (\%) } & \multirow[t]{2}{*}{ OR $(95 \% \mathrm{Cl})$} \\
\hline & TSH $0.3-2.5 \mathrm{mIU} / \mathrm{L}$ & TSH $2.5-4.8 \mathrm{mIU} / \mathrm{L}$ & $\mathrm{TSH}>4.8 \mathrm{mlU} / \mathrm{L}$ & \\
\hline Female & $232(25.41)$ & $163(30.66)$ & $58(36.02)$ & $1.27(1.09-1.49)^{*}$ \\
\hline Male & $212(38.90)$ & $77(49.68)$ & 7 (33.33) & $1.25(0.94-1.67)$ \\
\hline
\end{tabular}

${ }^{*} \mathrm{p}<0.01$. OR: odds ratio; TSH: thyroid-stimulating hormone; $\mathrm{Cl}$ : confidence interval

Table IV. ANOVA results for blood pressure in females with euthyroidism and subclinical hypothyroidism.

\begin{tabular}{|c|c|c|c|c|}
\hline \multirow[t]{2}{*}{ Parameter } & \multicolumn{2}{|c|}{ Mean \pm SD } & \multirow[t]{2}{*}{ F-value } & \multirow[t]{2}{*}{ p-value } \\
\hline & Euthyroidism (n = 1, 461) & Subclinical hypothyroidism $(\mathrm{n}=161)$ & & \\
\hline SBP (mmHg) & $122 \pm 17$ & $125 \pm 18$ & 4.33 & 0.038 \\
\hline $\mathrm{DBP}(\mathrm{mmHg})$ & $78 \pm 10$ & $81 \pm 10$ & 9.75 & 0.002 \\
\hline MAP (mmHg) & $93 \pm 11$ & $96 \pm 11$ & 8.26 & 0.004 \\
\hline
\end{tabular}

DBP: diastolic blood pressure; MAP: mean arterial pressure; SBP: systolic blood pressure

in our study consisted of middle-aged and elderly adults, De Pergola et al's study used obese adults ${ }^{(17)}$ and Walsh et al's study included young people. ${ }^{(6)}$ This difference in enrollment criteria may in part explain the disparity in the results. Another possible reason is population-specific heterogeneity. For example, Boucai and Surks' study showed that serum TSH concentrations were different between whites and blacks. ${ }^{(9)}$ The present study used a Chinese sample, while the studies by De Pergola et $\mathrm{al}^{(17)}$ and Walsh et $\mathrm{al}^{\left({ }^{(16)}\right.}$ used Caucasian samples. It is thus possible that the genetic differences between Chinese and Caucasians contributed to the discrepancy in the results. Furthermore, differences in iodine intake in different regions of the world or within the same country may also affect the results of these studies. Part of the results of Liu et al's research, (11) which was conducted on a population aged 18-85 years in northeast China, was not in accordance with the results of the present study. In Liu et al's study, the prevalence of hypertension was significantly higher in females from the subclinical hypothyroid group than in those from the euthyroid group, but variations of TSH within normal range did not affect blood pressure. ${ }^{(11)}$ This minor disparity may be due in part to different enrollment criteria and a difference in iodine intake between the northern and southern regions of China.

Prior to adjustment for the effects of the covariates BMI and WHR, HOMA-IR was associated with both TSH and blood pressure. However, after adjustment, these associations were no longer found to be significant, in line with previous studies. ${ }^{(17,18)}$ This finding implies that obesity-associated anthropometric indices, such as BMI and WHR, may have some confounding effect on the relationship between TSH and insulin resistance. Previous studies have also suggested that peripheral resistance to thyroid hormone may be present in individuals with obesity or insulin resistance, and that the control of TSH secretion by free thyroid hormones is possibly impaired in obesity. ${ }^{(14,17)}$ Thus, different levels of obesityassociated anthropometric indices in the studied populations may have induced the aforementioned inconsistent results regarding the association analysis between HOMA-IR and

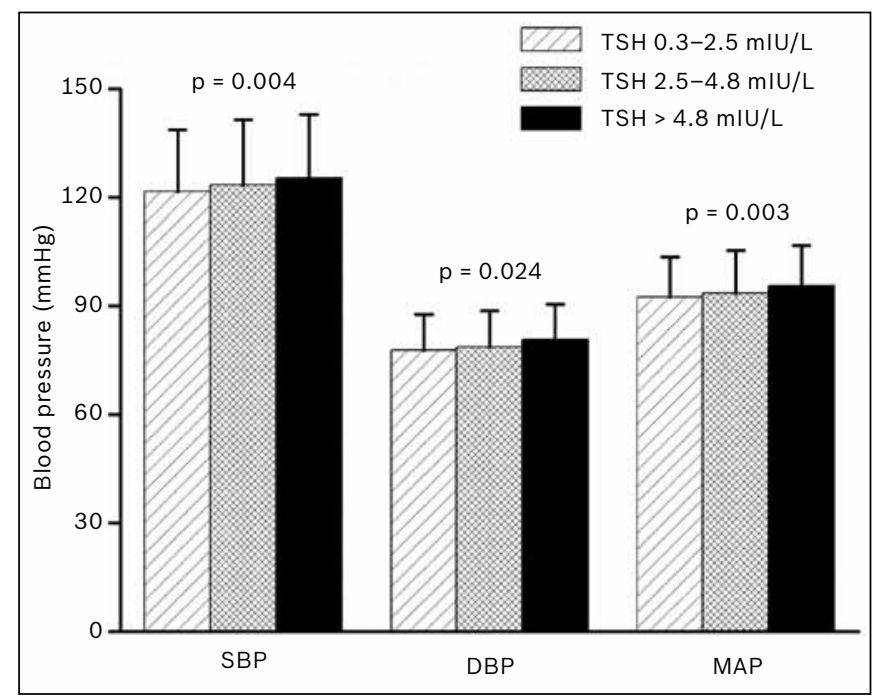

Fig. 1 Graph shows the blood pressure of female patients with different TSH ranges. DBP: diastolic blood pressure; MAP: mean arterial pressure; SBP: systolic blood pressure; TSH: thyroidstimulating hormone.

TSH, before and after adjustment for BMI and WHR. Therefore, it can be speculated that the improved insulin resistance observed during weight loss, especially the amelioration of central obesity, may help to lower blood pressure.

There are some limitations in the present study. First, the sample size of the male group was relatively small. This may have led to low statistical power in finding a significant result in the correlation analysis between TSH and blood pressure in males. Second, instead of serum lipid profiles, the history of hyperlipidaemia in the cohort was analysed. This may have resulted in the loss of some information in the present study. These important variables should be included in any future research.

In conclusion, the present study provides evidence of the detrimental effects of subclinical hypothyroidism and high normal TSH on hypertension. It will therefore be beneficial if more attention were given to the management of cardiovascular disease in patients with these characteristics, especially if they are elderly and female. 


\section{ACKNOWLEDGEMENTS}

We are very grateful to all the volunteers who participated in this study. This study was partially supported by the National Natural Science Foundation of China (30900699).

\section{REFERENCES}

1. Danzi S, Klein I. Thyroid hormone and blood pressure regulation. Curr Hypertens Rep 2003; 5:513-20.

2. Klein I, Danzi S. Thyroid disease and the heart. Circulation 2007; 116:1725-35.

3. Asvold BO, Bjøro T, Nilsen TI, Vatten LJ. Association between blood pressure and serum thyroid-stimulating hormone concentration within the reference range: a population-based study. J Clin Endocrinol Metab 2007; 92:841-5.

4. Ruhla S, Weickert MO, Arafat AM, et al. A high normal TSH is associated with the metabolic syndrome. Clin Endocrinol (Oxf) 2010; 72:696-701.

5. Taddei S, Caraccio N, Virdis A, et al. Impaired endothelium-dependent vasodilatation in subclinical hypothyroidism: beneficial effect of levothyroxine therapy. J Clin Endocrinol Metab 2003; 88:3731-7.

6. Walsh JP, Bremner AP, Bulsara MK, et al. Subclinical thyroid dysfunction and blood pressure: a community-based study. Clin Endocrinol (Oxf) 2006; 65:486-91.

7. Kawamoto R, Kohara K, Tabara $\mathrm{Y}$, et al. Insulin resistance and prevalence of prehypertension and hypertension among communitydwelling persons. J Atheroscler Thromb 2010; 17:148-55.

8. Sung KC, Kim BJ, Kim BS, et al. In normoglycemic Koreans, insulin resistance and adipocity are independently correlated with high blood pressure. Circ J 2004; 68:898-902.
9. Boucai L, Surks MI. Reference limits of serum TSH and free T4 are significantly influenced by race and age in an urban outpatient medical practice. Clin Endocrinol (Oxf) 2009; 70:788-93.

10. Wartofsky L, Dickey RA. The evidence for a narrower thyrotropin reference range is compelling. J Clin Endocrinol Metab 2005; 90:5483-8.

11. Liu D, Jiang F, Shan Z, et al. A cross-sectional survey of relationship between serum TSH level and blood pressure. J Hum Hypertens 2010; 24:134-8.

12. Fommei $\mathrm{E}$, lervasi $\mathrm{G}$. The role of thyroid hormone in blood pressure homeostasis: evidence from short-term hypothyroidism in humans. J Clin Endocrinol Metab 2002; 87:1996-2000.

13. Dickey RA, Wartofsky L, Feld S. Optimal thyrotropin level: normal ranges and reference intervals are not equivalent. Thyroid 2005; 15:1035-9.

14. Fernández-Real JM, López-Bermejo A, Castro A, Casamitjana R, Ricart W. Thyroid function is intrinsically linked to insulin sensitivity and endothelium-dependent vasodilation in healthy euthyroid subjects. J Clin Endocrinol Metab 2006; 91:3337-43.

15. Iqbal A, Figenschau $Y$, Jorde R. Blood pressure in relation to serum thyrotropin: The Tromsø study. J Hum Hypertens 2006; 20:932-6.

16. Gumieniak O, Hurwitz S, Perlstein TS, et al. Aggregation of high-normal thyroid-stimulating hormone in hypertensive families. J Clin Endocrinol Metab 2005; 90:5985-90.

17. De Pergola G, Ciampolillo A, Paolotti S, Trerotoli P, Giorgino R. Free triiodothyronine and thyroid stimulating hormone are directly associated with waist circumference, independently of insulin resistance, metabolic parameters and blood pressure in overweight and obese women. Clin Endocrinol (Oxf) 2007; 67:265-9.

18. Park HT, Cho GJ, Ahn KH, et al. Thyroid stimulating hormone is associated with metabolic syndrome in euthyroid postmenopausal women. Maturitas 2009; 62:301-5. 